Nardout-Lafarge, Élisabeth, « Procédés toponymiques chez Michon, Millet et Bergounioux », dans Yves Baudelle et Élisabeth Nardout-Lafarge (dir.), Nom propre et écritures de soi, Montréal, Les Presses de l'Université de Montréal, 2011, p. 122-135. 


\title{
Procédés toponymiques chez Michon, Millet et Bergounioux
}

\author{
Élisabeth Nardout-Lafarge
}

Dans la perspective d'un réexamen du rôle du nom propre dans «le pacte autobiographique ", quelle place faire à ces autres noms que sont les toponymes? Exception faite, bien sûr, de quelques textes canoniques - au premier rang desquels "Noms de pays: le nom ${ }^{1}-$, qui constituent eux-mêmes des théories littéraires du nom propre, le toponyme, objet privilégié de l'onomastique historique, reste le parent pauvre des études sur le nom propre littéraire, qui ne le retiennent généralement qu'au titre de la mimesis, comme l'un des effets de réel les plus attestables, marqueur d'un espace référentiel soit familier, soit dépaysant. Parallèlement, les études sur le biographique, qu'elles portent sur les biographies, les autobiographies ou les autofictions, s'interrogent moins sur le lieu que sur le milieu ou la famille du sujet dont l'histoire est racontée.

Le déploiement des toponymes dans les textes «obliquement autobiographiques " ${ }^{2}$ de Pierre Michon, Richard Millet et Pierre Bergounioux produit des effets d'écho entre le lieu de naissance de l'auteur, tel qu'il apparaît conventionnellement dans le paratexte, et les toponymes que le texte donne à lire. S'ils exploitent cette résonance des noms entre eux, ces textes ne reconduisent pas sans les mettre à l'épreuve ces associations implicites entre les noms propres qui balisent l'identité. Dans

I. Marcel Proust, «Noms de pays: le nom», dans À la recherche du temps perdu, éd. Jean-Yves Tadié, Paris, Gallimard, «Bibliothèque de la Pléiade», t. I, I987, p. 376-420.

2. Selon l'expression de Jean-Pierre Richard, "Servitude et grandeur du minuscule», dans L'état des choses, Paris, Gallimard, I990, p. 87. 
le vaste ensemble de l'écriture autobiographique contemporaine, Dominique Viart caractérise Vies minuscules de Michon ${ }^{3}$ et une bonne partie de l'œuvre de Bergounioux comme des «récits de restitution» et des «essais-fictions" hantés par la littérature et la critique ${ }^{4}$; les romans et récits du cycle corrézien de Millet peuvent également entrer dans cette catégorie.

Ces trois ouvres interrogent très spécifiquement l'intrication du biographique et du lieu. C'est l'hypothèse de l'essai que leur consacre Sylviane Coyault-Dublanchet, La province en héritage: «Sans doute, le récit de mémoire requiert-il plus que tout autre genre narratif une forte présence du lieu " . À des degrés variables en effet, Michon, Bergounioux et Millet élaborent une sorte de fantasme de l'origine, générateur de fictions dont la reprise, les déplacements, les variations constituent un enjeu central de leurs poétiques respectives. Dans La cécité d'Homère de Pierre Bergounioux, une phrase aux allures d'axiome l'affirme catégoriquement: "On est le lieu de la terre où l'on naît " ${ }^{6}$; la formule est reprise et précisée dans Le chevron: "Car on est les choses auxquelles on naît ${ }^{7}$.

\section{Le lieu}

Le lieu que ces écrivains ont en commun est d'abord référentiel, c'est le Limousin. C’est la Creuse de Pierre Michon, né «dans la Creuse» (on notera que "dans", plus que «en", associe le lieu à une matérialité) selon la très brève notice biographique de Vies minuscules qui tait le nom du hameau natal, «Les Cards», révélé dans les entretiens avec l'auteur et

3. Pierre Michon, Vies minuscules (1984), Paris, Gallimard, «Folio», I996. Désormais: $V M$.

4. Dominique Viart, "Essais-fictions: les biographies (ré)inventées», dans Marc Dambre et Monique Gosselin-Noat (dir.), L'éclatement des genres au $\mathrm{XX}^{e}$ siècle, Paris, Presses de la Sorbonne Nouvelle, 200I, p. 33I-345, et "Les "fictions critiques" de Pierre Michon", dans Agnès Castiglione (dir.), Pierre Michon. L'écriture absolue. Actes du premier colloque international Pierre Michon, Saint-Étienne, Presses de l'Université de Saint-Étienne, 2002, p. 203-219.

5. Sylviane Coyault-Dublanchet, La province en héritage. Pierre Michon, Pierre Bergounioux, Richard Millet, Genève, Droz, 2002, p. I8I. $\mathrm{CH}$.

6. Pierre Bergounioux, La cécité d'Homère, Strasbourg, Circée, 1996, p. I8. Désormais:

7. Pierre Bergounioux, Le chevron, Lagrasse, Verdier, 1996, p. 7. 
présent dans le récit. Ce sont les Corrèze, celle de Bergounioux, né à Brive, avec ses deux côtés, la petite ville engoncée et l'échappée vers le Quercy, et celle de Millet, né à Viam, aux confins du plateau de Millevaches. Ainsi, dès le paratexte, le toponyme fait sens de manière différente selon une hiérarchie qui rappelle s'il était besoin que tous les lieux ne sont pas égaux: la ville, autosuffisante - fût-elle souspréfecture -, le village, qui a besoin de la précision du département dans lequel il se trouve, et le lieu-dit, radicalement privé de pouvoir référentiel et donc tu. Le lieu géographique est évidemment aussi paysage mental et social, "matériau mémoriel et imaginaire " ${ }^{8}$, écrit Sylviane Coyault-Dublanchet, qui le synthétise pour les trois auteurs dans une "conscience malheureuse de provincial " qu'elle impute "au discrédit attaché aux valeurs paysannes $»^{10}$. Les trois œuvres réitèrent l'écart de la province, l'éloignement, la relégation, l'étroitesse d'esprit proportionnelle à l'exiguïté des lieux, le retard, sinon le désert culturel. Pour ces auteurs, l'isolement se mesure à ses effets sur les gens et sur les choses ainsi que sur leur propre parcours d'écrivain. Ils adoptent sur le lieu une position éthique assez semblable. Comme ils le font pour les petites gens et les ancêtres, réels et imaginaires, ils rendent aux lieux qu'ils évoquent la dignité littéraire d'un paysage poétique qui fait fonds, non sur le pittoresque de l'éloignement, mais sur l'angoisse de la déshérence et de la mort.

La démarche est cependant trop consciente d'elle-même pour ne pas être en même temps mise à distance, car l'héritage provincial est aussi, et peut-être d'abord, un héritage littéraire. Comme le montre Alain $\operatorname{Corbin}^{11}$, la littérature invente la province dans le paradoxe. D'une part, la province est contraire à la littérature, elle lui est même néfaste, leurs territoires ne peuvent pas coïncider, sauf à consentir à l'adjectivation régionaliste. Pierre Bergounioux définit sa région comme l'un de "ces univers que leur subordination ou leur éloignement vouait à une

8. La province en héritage, op. cit., p. 264-265.

9. Ibid., p. 263-264.

Io. Ibid., p. 20.

II. Alain Corbin, "Paris-Province», dans Pierre Nora (dir.), Les lieux de mémoire, t. II, Paris, Gallimard, «Quarto», 1997, p. $285 \mathrm{I}-2888$. 
sorte d'inexistence culturelle, de vide symbolique $»^{12}$ et insiste sur «le divorce entre la vie qu'on menait et celle que décrivaient les livres " ${ }^{13}$. D'autre part, la province est un topos de la littérature, le mot même agissant comme embrayeur intertextuel et suggérant de multiples scènes de Balzac, Mauriac, Chardonne ou Giraudoux. La construction littéraire reprend et renforce les clichés, et Bergounioux rappelle volontiers, notamment dans Le premier mot ${ }^{14}$, la genèse textuelle d'un certain Limousin littéraire, de l'escolier de Rabelais au croquant de Sartre en passant par Monsieur de Pourceaugnac. Dès l'incipit de «Vie d'André Dufourneau ", la première des Vies minuscules, la province s'inscrit sur le mode privatif par l'évocation d'un déficit tant géographique que littéraire: «La province dont je parle est sans côtes, plages ni récifs; ni Malouin exalté ni hautain Moco n'y entendit l'appel de la mer...» (VM, p. I3). L'appréhension du lieu passe par une sorte d'intériorisation de sa dépréciation, spécifiquement de sa disqualification littéraire: "Tous ont le sentiment d'évoquer des contrées qui n'ont pas eu comme la Touraine leur Balzac, comme la Provence leur Giono, ni même comme la Bretagne leur Chateaubriand », commente Sylviane CoyaultDublanchet ${ }^{15}$. Mais comment écrire le lieu dès lors que Touraine, Bretagne et Provence s'offrent désormais quasi équivalentes à la perception contemporaine, traversées, comme le dit Marc Augé, par ces autoroutes toutes semblables qui évitent les agglomérations tout en signalant leurs attractions sur des panneaux géants ${ }^{16}$ ? Cette uniformisation de l'expérience du lieu interdit à l'écrivain d'aujourd'hui de s'en faire le chantre attitré. Ni Bergounioux, ni Michon, ni Millet n'aspirent à l'identification entre l'auteur et son lieu d'origine. Sur ce plan d'ailleurs, comme le note aussi Sylviane Coyault-Dublanchet, la filiation littéraire

I2. Pierre Bergounioux, «Une prose noire», L’Eil de boeuf, nº II, p. 2I, cité dans La province en héritage, op. cit., p. 76.

I3. Pierre Bergounioux, Chasseur à la manque, Paris, Gallimard, "Le promeneur", 20IO, p. 31.

I4. Pierre Bergounioux, Le premier mot, Paris, Gallimard, 200I, p. 49. Désormais: PM.

15. La province en héritage, op. cit., p. 179. Sylviane Coyault-Dublanchet rappelle également qu'au contraire des membres de "l'école de Brive», ces trois écrivains "ne sont pas prophètes en leur pays et suscitent parfois une vague hostilité chez les lecteurs limousins" (p. I84).

I6. Marc Augé, Non-lieux. Introduction à une anthropologie de la surmodernité, Paris, Seuil, «La librairie du XXI ${ }^{\text {e }}$ siècle», I992, p. I22-I23. 
est tout autre, et l'un des principaux intercesseurs revendiqué par Bergounioux et Michon est Faulkner, que Michon cependant identifie à l'éloignement provincial: "plouc de ce ramas de ploucs, un plouc du Sud $»^{17}$.

Ces œuvres construisent avec le lieu un rapport imaginaire complexe. D’une part, ces écrivains, nés dans un lieu réputé contraire à la littérature, acceptent ou feignent d'accepter ce postulat pour mieux le réfuter, y entrant par ce qui les en sépare; c'est l'hypothèse de Pierre Bergounioux dans son article sur Pierre Michon, repris dans La cécité d'Homère: "Son ouvre, et la qualité singulière de celle-ci, procèdent de sa relégation extrême» $(\mathrm{CH}, \mathrm{p} .76)$. D'autre part, leur travail sur ce lieu (sur la pauvreté duquel tous trois s'accordent - «Rien ou presque, c'est ce que quiconque a vu le jour vers le milieu du siècle au milieu du pays touchait par la force des choses", écrit aussi Bergounioux $(\mathrm{CH}$, p. 76-77) - s'effectue moins à partir de ses particularités que du point de vue de sa disparition. Le lieu, avec ses noms, est thématisé et plus encore analysé à partir de sa perte.

\section{Michon: les noms pauvres}

Les nombreux commentateurs de Michon ont souligné son goût pour les noms propres. Lisant, dans Rimbaud le fils ${ }^{18}$, l'itinéraire ardennais du poète, Jean-Pierre Richard évoque «la puissance littérale des toponymes $»^{19}$. Bruno Blanckeman parle lui, "du chant des noms propres $»^{20}$. Dans son étude de Vies minuscules, Dominique Viart insiste aussi sur la répétition des toponymes qui, selon lui, «[...] loin de particulariser des lieux, [...] s'universalisent dans une évocation du pays profond $»^{21}$. Sylviane Coyault-Dublanchet montre comment la géographie biographique de Vies minuscules oppose des toponymes minuscules qui balisent

I7. Pierre Michon, "Le père du texte», dans Trois auteurs, Lagrasse, Verdier, I997, p. $79-88$, p. 85 . $R F$.

18. Pierre Michon, Rimbaud le fils (I99I), Paris, Gallimard, «Folio», I993. Désormais:

19. Jean-Pierre Richard, "Pour un Rimbaud", dans Terrains de lecture, Paris, Gallimard, 1996, p. 33-34.

20. Bruno Blanckeman, "Pierre Michon: une poétique de l'incarnation", dans Pierre Michon. L'écriture absolue, op. cit., p. I45-I5I.

2I. Dominique Viart, "Vies minuscules» de Pierre Michon, Paris, Gallimard, «Foliothèque», 2004, p. 62. 
l'espace familial - Mourioux, signifiant maternel, où se trouvent «Les Cards» et "la maison d'école», Mazirat où vivent les grands-parents paternels - aux toponymes majuscules porteurs du vaste monde ${ }^{22}$, essentiellement l'Afrique, qui sera la "vocation» d'André Dufourneau, et l'Amérique où l'ivrogne Fiéfié Décembre situe un Antoine Péluchet fictif qui se substitue au vrai.

La remotivation poétique du toponyme réel obéit à la logique propre du texte. Ainsi, l'énumération de ces noms inconnus d'une ruralité qu'aucune histoire n'a mise en lumière est sensiblement différente dans Rimbaud le fils et dans Vies minuscules. Là où les "grands pas» du poète ont passé, quelque chose de son éclat demeure: «[...] des patelins formidables et mornes comme des coups de canon, des mouchoirs de poche enfoncés dans la bouche: Warcq, Voncq, Warnécourt, Pussemange, Le Theux» (RF, p. 59). On ne trouvera pas cette force dans les noms répétés à plusieurs reprises dans Vies minuscules: "Chatelus, Saint-Goussaud, Mourioux» (passim). Faute d'un Rimbaud qui les rende «formidables», les «patelins» ne sont que «mornes», voire morts. L'itinéraire qu'ils balisent est celui de bistrots où des ivrognes s'abîment jusqu'à l'hébétude, ou celui de cimetières où reposent les "minuscules" dont le narrateur raconte les vies. Aussi la morphologie des toponymes s'en trouve-t-elle proportionnellement réinterprétée. Si, comme l'écrit Jean-Pierre Richard, "Warcq, Voncq, Warnécourt, Pussemange, Le Theux [...] canonnent, tout autant qu'ils asphyxient, ou qu'ils étranglent " $^{23}$, "Chatelus, Saint-Goussaud, Mourioux» n'ont pas cette énergie sonore. Ces trois noms, toujours les mêmes et inscrits dans le même ordre, n'évoquent pas la marche ou la fuite, ils disent au contraire l'enfermement dans un même circuit. Ils réitèrent la banalité irrémédiable d'un lieu devant lequel la narration s'avoue incapable d'anoblissement poétique.

Ce ne sont pas pour autant des noms ridicules, mais seulement des noms ordinaires, des noms pauvres, éloignés de toute distinction: Chatelus n'est même pas un château, Saint-Goussaud, "saint naïf et délaissé» (VM, p. 69), rappelle l'épithète dépréciative "rougeaud» que le stéréotype accole volontiers au paysan; dans Mourioux enfin, s'entend

22. La province en héritage, op. cit., p. I78-I79.

23. Jean-Pierre Richard, "Pour un Rimbaud", op. cit., p. 34. 
une version patoisante de la mort, car les noms gardent trace du patois dans la langue de l'école et de la République. La triade d'adjectifs qui, à la fin du texte, qualifie le père suicidé accroché à l'image de son fils (et qui pourrait s'appliquer aussi bien aux ivrognes harangués par Fiéfié Décembre) paraît répondre de manière également symétrique aux trois toponymes, comme eux «innocents, balourds et consubstantiels» (VM, p. 68).

On observe une autre stratégie de cette déflation du nom dans des formules plus ou moins laconiques, parfois implicitement exclamatives, par lesquelles le toponyme local vient barrer la possibilité de récit: "Que dire d'une enfance au Châtain?»(VM, p. 40). À l'opposé de Guermantes, dont les syllabes orangées s'offraient au dépliement, le hameau minuscule au nom minuscule - dont la couleur brune de la châtaigne évoque le Limousin et la pauvreté paysanne - n'enclencherait aucun récit. Dénégation feinte, bien sûr, sur laquelle repose toute l'entreprise qui consiste à écrire ces vies. Dans "Vie des frères Bakroot", le toponyme vient renforcer le divorce entre l'univers des livres - le Kipling richement illustré offert par Achille - et celui de leurs lecteurs, le vieux professeur contrefait et son élève, l'aîné des Bakroot:

[...] au premier plan, rêveurs, rasés, courtois et rapaces, des gentlemen et des fripouilles, galonnés, indiscernables sous la même vareuse écarlate et le casque parfait de l'armée des Indes, contemplaient calmement ce monde, Himalayas, rois barbus et laidies pulpeuses sous l'ombrelle, ce monde qui était leur pâture (Pauvre Achille, pâture du monde, qu'est-ce que tout cela pouvait bien lui dire? Et au fils Bakroot, de Saint-PriestPalus?) (VM, p. II5)

Ainsi renvoyé à la toute fin de la parenthèse, le toponyme porte la marque ultime de l'éloignement de la littérature, de la relégation, et ce dispositif a pour effet de donner en retour à sa morphologie une charge de trivialité. La même incongruité est soulignée quelques pages auparavant: "Saint-Priest-Palus, dont ils étaient à peine sortis, sur le plateau rocheux vers Gentioux, sous le toit d'une ferme pauvre de cette terre vaine [...] y lire Salammbô était inexplicablement comique»(VM, p. 107). Le grotesque tueur de littérature qui fait que Péluchet, trop Pécuchet, ne sera pas Rimbaud, n'est pas inscrit par avance dans les noms, il s'y trouve ajouté par le soulignement du dérisoire, qui désamorce les pratiques conventionnelles de l'anoblissement littéraire. Mais 
il s'agit sans doute moins de priver ces noms humbles de tout potentiel poétique que de rappeler le fonds de pauvreté dans lequel le narrateur les puise. Ce constant détrônement du nom ne cherche donc pas à être efficace; l'écriture de Vies minuscules maintient plutôt une tension entre la langue lyrique et cultivée du narrateur et l'humilité du toponyme obscur qui y demeure étranger et continue d'y faire tache. Pas plus que la mort des minuscules, la pauvreté des noms n'est transfigurée par la littérature, mais seulement prise en charge, assumée.

\section{Millet: le mythe dans le nom}

La transposition de "Viam» le village natal, en "Siom», centre de la géographie fictive dans tous les textes corréziens de l'auteur ${ }^{24}$, constitue un exemple révélateur de l'usage du toponyme chez Millet. Siom a donc le rôle d'une matrice fictionnelle qui donne vie à la saga des Pythre et à celle de leurs voisins, il fonctionne comme un réservoir de figures, de destins, de tragédies, de noms. Siom signifie également en ce que la transposition de Viam y reste lisible et attache ainsi, par le lieu, la fiction à la biographie. Siom pour Viam est d'autant plus significatif qu'il constitue le seul exemple de fabrication d'un toponyme fictif dans ce pan de l'œuvre, les autres étant repris au répertoire onomastique régional, où les finales en -eat, -ac, -eix, -is font signe vers le Massif central, l'Auvergne: Meymac, Bugeat, Villevaleix, Mont-Gardis, etc. Or Viam n'appartient pas à ce répertoire et fait partie de ces noms, finalement assez nombreux, qui prennent en défaut les lois de la motivation ethnogéographique dont Yves Baudelle a montré la rentabilité pour la logique de la vraisemblance dans le

24. Outre la trilogie composée de La gloire des Pythre (Paris, P.O.L., I995; Gallimard, "Folio", 1997. Désormais: GP), L'amour des trois sæurs Piale (Paris, P.O.L., I997; Paris, Gallimard, "Folio», 1999) et Lauve le pur (Paris, P.O.L., I999; Paris, Gallimard, "Folio», 2004), cet ensemble de textes centrés sur le village de Siom comprend également des récits: Le renard dans le nom (Paris, Gallimard, 2003. Désormais: $R N$ ), Le cavalier siomois (Brive, F. Janaud, I999; Paris, La Table ronde, 2004), Tarnac (Paris, Gallimard, "L'arpenteur", 20IO), ainsi que des romans autobiographiques ou autofictifs, Ma vie parmi les ombres (Paris, Gallimard, 2003. Désormais: MVO), Dévorations (Paris, Gallimard, 2006; "Folio», 2008). Signe du rôle matriciel qu'il joue dans l'œuvre, Siom apparaît même hors de la fiction dans L'art du bref (Paris, Gallimard, "Le cabinet des lettrés», 2006), récit de la vie d'un photographe ambulant réel. 
roman réaliste ${ }^{25}$. Sensible aux noms rares - qu'on pense aux anthroponymes Pythre, Piale, Lavolps ou Lauve (qui est en fait un toponyme) -, Millet fait entendre dans cette singularité qui, non seulement distingue celui qui le porte, mais le plus souvent l'isole et le stigmatise, une sorte d'irréductible noyau de propre. L'étrangeté de ces noms, souvent commentée par le narrateur, tient aussi à ce que, de moins en moins portés, ils ne sont plus prononcés que par de rares locuteurs et ne seront bientôt que des inscriptions sur des tombes. Sylviane Coyault-Dublanchet souligne l'obsession des cimetières commune à Millet et à Bergounioux. Et dans Vies minuscules, Michon évoque aussi ces noms qui s'éteignent: "[...] quand le nom cesse de s'apparier à des vivants. Seules le profèrent encore des bouches sans langue» (VM, p. 68). De même, chez Millet, c'est de n'être plus habités que les noms sont étranges, justement parce qu'ils ne sont plus que des noms. Donner forme aux récits de Viam, c'est donc réactiver les noms par des fictions et combler ce défaut de sens d'un nom qui n'est plus "apparié»: «[...] tous les récits [...] s'oublient [...] sauf les noms propres, lesquels durent généralement plus longtemps que les corps et que le souvenir» $(R N, \mathrm{p}$. I3).

Millet accentue encore la rareté de Viam dans une transposition qu'il charge d'une dimension mythique, car dans Siom, bien sûr, trône Sion: "[...] ce Siom que, répétons-le, nous prononcions Sion, et qui avait pour nous quelque chose de céleste que le curé tentait de nous représenter mais à quoi nous ne comprenions pas grand-chose, sinon que, damnés ou innocents, pauvres et moins pauvres, nous vivions en terre bénie» (GP, p. IIo). Cependant, comme dans Vies minuscules, le nom cristallise une tension. D'une part, le toponyme se trouve magnifié par l'évocation de la Jérusalem céleste, et son pouvoir de sacralisation s'étend en retour à l'ensemble du système onomastique du texte. L'inflexion biblique du nom Siom affecte tous les noms: des plus rares aux plus humbles, des toponymes que l'usage fait oublier aux patronymes que des connotations grotesques rendent impossibles à porter, tous tendent vers le mythe. Le nom transposé impose par la référence biblique une sorte d'exemplarité, et le déclin de Siom, bourgade banalement victime de la désertification rurale, prend dès lors valeur de

25. Voir Yves Baudelle, Sémantique de l'onomastique romanesque, Thèse de doctorat (1989, Université Paris 3), 2 vol., 990 p. 
fable, de fin d'un monde, le Plateau de Millevaches devenant Terre de Caïn. Mais, d'autre part, cette mythification entre en conflit, dans l'œuvre même, avec la trivialité de l'expérience du lieu. Malgré les efforts pédagogiques du curé, le mythe de Sion inscrit dans Siom échappe à ses habitants, et l'enfoncement dans la terre connotée par l'excrément s'oppose cruellement à l'allusion céleste. La transposition de Viam en Siom marque cette tension, par l'excès, la disproportion qu'elle donne à lire comme un pari perdu d'avance. Seule la mort qui fait du lieu un infini cimetière autorise Viam à devenir Siom ${ }^{26}$. Ce que le procédé reverse dans la fiction, c'est donc moins la morphologie d'un nom que le pouvoir d'évocation, l'embrayeur d'imaginaire dont cette morphologie est garante pour un sujet donné, comme l'ont montré les travaux d'Eugène Nicole ${ }^{27}$. En ce sens, parce qu'elle explicite les récits que ce nom contient virtuellement, la transposition paraît d'abord excédentaire. Siom laisse néanmoins perdre certaines des potentialités imaginaires de Viam, notamment la connotation exotique due aux sonorités quasi asiatiques de ce «Viam» pourtant authentiquement corrézien. Entre Viam et Siom s'entend en effet le relais de "Siam», souvenir des anciens atlas et de la géographie coloniale que Ma vie parmi les ombres réinscrit sans doute dans le nom "Lam», attribué à un personnage trouble au destin tragique, «métis d'Indochinois et d'Européen " que Jacques Champetiers, ancien de Diên Biên Phu, ramène en Corrèze ( $M V O$, p. I90-I9I).

La transposition fonctionne donc comme une signature, chiffre d'une écriture ainsi explicitement nouée à un lieu qu'elle donne à la fois pour sien, natal, et disparu. Siom pour Viam signe aussi une manière littéraire liée au secret et à l'énigme, procédé parallèle à cet autre effet de signature qu'est la mention des Millet homonymes de l'auteur, le musicien et surtout le peintre, auteur d'un célèbre Angélus emblématique de la ruralité et que Richard Millet se réapproprie pour intituler

26. Il est également possible de lire dans cette transposition l'inscription de filiations littéraires elles aussi antithétiques, avec Barrès dont "la colline inspirée» se trouve à Sion-Vaudémont, comme le note Jean-Yves Laurichesse dans Richard Millet. L'invention du pays (Amsterdam/New York, Rodopi, «Faux titre», 2007, p. 184), et avec Gracq, qui évoque, notamment dans Carnet du grand chemin (dans Euvres complètes, Paris, Gallimard, «Bibliothèque de la Pléiade», t. II, I995, p. IOIO), ses séjours à Sion en Vendée. 27. Eugène Nicole, «L'onomastique littéraire», Poétique, nº 54, avril I983, p. 233-253. 
l'un de ses récits ${ }^{28}$. Ma vie parmi les ombres réinscrit ce hasard de l'homonymie qui désordonne les généalogies et offre un nom déjà illustre, propulsant vers la gloire tout en condamnant à l'inévitable imposture, comme il en est de Viam devenu Siom: le narrateur qui s'appelle Pascal Bugeaud se rêve une fausse parenté avec le maréchal Bugeaud, limousin lui aussi. La transposition du toponyme, qui s'accompagne de l'invention du gentillet «Siomois», dit bien cette gémellité tendue de la fiction avec la vie.

\section{Bergounioux: de la collection des noms à l'absence de nom}

Trois pratiques du toponyme se croisent dans cette œuvre qui s'applique, de récit en récit, à élucider «ce qu'une époque, un endroit [...] pouvaient jeter de sombre et d'amer dans une âme $»^{29}$. La collection est le premier usage du toponyme local. Collectionneur, glaneur (il évoque, dans $L a$ casse $^{30}$, ses collectes de métaux recyclés dont il fait des sculptures), Bergounioux s'intéresse aussi aux noms et à l'étymologie qui les ordonne, comme à l'entomologie, qu'il pratique, et à la géologie. Échantillons de roc, insectes rares et noms anciens sont ainsi les objets modestes d'une connaissance studieuse, patiente, qui renvoie aux instituteurs de campagne et aux érudits des sous-préfectures, tel le géologue Mouret "positiviste et barbichu", auteur d'un austère traité lu avec bonheur à la bibliothèque municipale $\left(P M\right.$, p. 88). Dans La ligne ${ }^{31}$, texte méditatif inspiré par l'eau (canaux, rivières, et étangs où pêcher à la ligne), deux pages sont consacrées aux toponymes corréziens qui comportent ce sème aquatique. Décryptés jusqu’à leur origine galloromaine ou celte et dûment classés dans une taxinomie où l'érudition étymologique bride la dérive poétique, «les vieux noms» viennent appuyer une explication physique, scientifique, au malaise obsédant qui s'attache au paysage: "La zone oblique, accidentée, elle aussi frontalière, qui sépare la plaine aquitaine du Massif Central, dont le cœur de

28. Richard Millet, L'angélus, Paris, P.O.L., 1988.

29. Pierre Bergounioux, Simples, magistraux et autres antidotes, Lagrasse, Verdier, 20oI, p. 47-48. Désormais: $S M A$.

30. Pierre Bergounioux, La casse, Saint-Clément-de-Rivière, Fata Morgana, 1994.

31. Pierre Bergounioux, La ligne, Lagrasse, Verdier, 1997. Désormais: L. 
granit, hermétique à l'eau la rejette en rigoles, en ruisseaux, transpire et se ressuie, nourrit des tourbières, produit des étangs" ( $L$, p. I3).

L'un de ces noms cependant, Brive, celui de la ville natale dont plusieurs récits élaborent la topographie intime, fait l'objet d'un traitement plus singulier et prend valeur d'emblème par le relais de son signifié: "Brive, sur la Corrèze, et son petit doublet, Brivezac, sur la Dordogne, c'est briva, le pont, en celtique comme aujourd'hui Brücke en allemand, bridge en anglais» ( $L$, p. I4). Cette étymologie est plusieurs fois reprise, notamment dans Simples, magistraux et autres antidotes, où le narrateur compte parmi les «alliés» qui l'aident à supporter «l'accablement» du lieu, "les ouvrages d'art»

[...] que les inégalités du terrain obligeaient à multiplier. La ville en retirait son nom prélatin, Brive comme bridge en anglais, Brücke en allemand. Ils étaient trois à enjamber la rivière dans les limites de l'agglomération, auxquels s'ajoutaient ceux de Malemort en amont, ceux de Grange. On lança sur le tard, celui des Baylies-Basses, en béton précontraint d'une seule travée. Ce n'est pas tout. Un grand viaduc permettait à la ligne de Toulouse de franchir la profonde coupure de Planchetorte qui séparait la cuvette du Causse du Martel tandis que des tunnels trouaient les remparts successifs de l'est et du nord. (SMA, p. 23-24)

Ces «ouvrages d'art» inscrivent dans le lieu le travail humain et la strate industrielle, qui y figurent aussi par l'évocation des véhicules les voitures surtout, longuement décrites, mais aussi les machines agricoles et les trains, dont les noms souvent anglais s'accrochent aux souvenirs d'une adolescence des années soixante. La casse, qui parachève en quelque sorte ce récit des choses et des machines, situe Bergounioux au plus près de François Bon (dont il a aussi commenté l'œuvre dans La cécité d'Homère [CH, p. 95-II6]). Le grouillement métallique qui envahit la ruralité et va la transformer jusqu'à sa disparition est évoqué sur le mode de la profusion plutôt que de l'agression. L'enthousiasme énumératif qui porte la phrase citée plus haut et accumule les exemples de "ponts» atteste l'importance du motif. Le pont certes relie, mais il sépare aussi, il désigne dans le paysage de "profonde[s] coupure[s]", d'autant qu'il peut être barrage, ou encore viaduc ferroviaire livrant passage aux «longs rapides verts olive» lancés à toute allure dans la campagne, objets de la fascination enfantine du narrateur (SMA, p. 33). Par là, Brive le pont s'apparente à ce «motif omniprésent 
de la blessure [...] des objets acérés, des formes déchirantes» qu'a relevé Jean-Pierre Richard ${ }^{32}$.

Les textes ont également recours à des stratégies d'esquive ou de substitution du toponyme, ce dont témoigne la fréquence dans l'œuvre de Bergounioux de désignateurs qui ne sont pas à proprement parler des toponymes (quoiqu'ils en aient les fonctions), mais des descriptions définies lexicalisées, comme cette "Nationale 20 ", axe central de la géographie des récits. Chez Bergounioux, les routes, qui partagent avec les ponts de nombreuses caractéristiques, jouent d'ailleurs un rôle important. Dans l'inscription du lieu natal, le toponyme est fréquemment concurrencé par des descriptions définies telles que "la maison rose», titre d'un roman autobiographique ${ }^{33}$, ou encore «la casse». Du nom propre, c'est en quelque sorte la majuscule, grandiose ou pittoresque, qui se trouve ainsi supprimée. En esquivant la toponymie qui relie le lieu à la loi, Bergounioux situe la géographie du texte dans une familiarité et une immédiateté, calque d'un usage plus quotidien du lieu, où l'on parle du haut de la rue, du bas de la côte, du magasin sur la droite, etc., sans recourir aux noms, inexistants, ignorés ou réservés à une extériorité. Cet évitement du nom tient aussi d'un mouvement de resserrement centripète du texte qui tente de saisir dans le lieu son aspect le plus intime et le plus précisément révélateur de ce qu'il est, jusqu'au caillou, au grain du roc.

Mais dans cette entreprise d'élucidation de ce qui, dans le lieu, explique l'expérience que le narrateur en fait, la précision ironiquement scientifique côtoie l'évanescence de l'impression, l'une et l'autre inaptes à tenir dans un nom. Le récit Univers préférables ${ }^{34}$, dont le titre dessine d'emblée un espace hors cadastre, est exemplaire de ce procédé: il rassemble les rêveries qu'ont inspirées au narrateur «une grande maison fermée $[. .$.$] par le fait même ouverte à toutes les spéculations»(UP, p. I2),$ située en bordure de la Nationale 20, au moment où l'enfant qui partait en vacances éprouvait comme une promesse et une autorisation le sentiment du changement de la Corrèze sombre en un Quercy lumineux.

32. Jean-Pierre Richard, "La splendeur, la blessure", dans L'état des choses, op. cit., I990, p. I07-I29 (p. 107).

33. Pierre Bergounioux, La maison rose, Paris, Gallimard, 1987.

34. Pierre Bergounioux, Univers préférables, Saint-Clément-de-Rivière, Fata Morgana, 2003. Désormais: UP. 
À la sortie d'un village, vacante, «la maison du tournant» marque ce lieu privilégié qui redonne un peu du temps enfui, et que, comme tous ces «ravins», "combes» et «talus» (ibid.), un nom ne saurait définir.

Que conclure de ces trois exemples trop rapidement parcourus? On notera que pour chacun des auteurs, c'est dans le registre toponymique que se négocie le pacte autobiographique, au risque d'ailleurs d'une surinterprétation critique de leur ancrage dans l'origine. En effet, Michon et Bergounioux reproduisent les toponymes réels alors qu'ils taisent les anthroponymes ${ }^{35}$; par la transposition fictive d'un seul toponyme réel, Millet pour sa part revendique le pacte romanesque, tout en inscrivant l'autobiographique à même cette transposition. La primauté du toponyme sur l'anthroponyme dans le procès d'identification du sujet confirme l'enjeu collectif de ces écritures où la communauté, non seulement régionale mais sociale et imaginaire (celle des «minuscules» chez Michon, des «croquants» chez Bergounioux, des "gourles» chez Millet), intervient dans la construction de soi. Dès lors l'identité apparaît comme une nébuleuse de noms dont aucun n'a le monopole du propre, et dont l'ensemble dessine une signature multiple, instable mais cohérente. La remotivation poétique des toponymes obéit à la logique de chaque récit. Michon en exhibe la pauvreté et les fait entrer, ainsi exposés, dans la "grande langue» par laquelle il entend les sauver. Bergounioux en creuse l'étymologie pour les faire contribuer à l'élucidation inquiète du lieu qu'il explore et qui les déborde finalement. Millet encrypte dans le nom transposé une ambition mythographique en même temps que ce qui lui résiste. Dans tous les cas, ces noms signifient moins par leur morphologie que par les dispositifs de leur énonciation.

35. La manière quasi accidentelle dont le nom "Michon" apparaît dans Vies minuscules, dans «Vie de la petite morte" à propos de la sœur morte et en référence à la grandmère paternelle ("Elle avait de grands yeux bleu sombre - venus de Clara assurément, Michon née Jumeau»; VM, p. 238), a été commentée notamment par Dominique Viart ("Vies minuscules» de Pierre Michon, op. cit., p. 60-6I). 